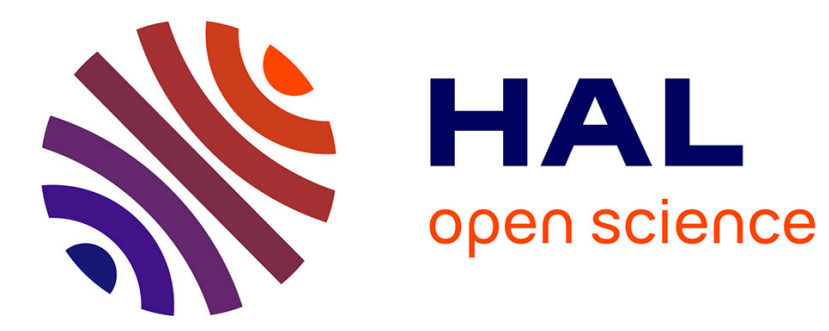

\title{
Iterative Closest Curve: a Framework for Curvilinear Structure Registration Application to 2D/3D Coronary Arteries Registration
}

Thomas Benseghir, Grégoire Malandain, Régis Vaillant

\section{- To cite this version:}

Thomas Benseghir, Grégoire Malandain, Régis Vaillant. Iterative Closest Curve: a Framework for Curvilinear Structure Registration Application to 2D/3D Coronary Arteries Registration. MICCAI Medical Image Computing and Computer Assisted Intervention, Sep 2013, Nagoya, Japan. pp.179-186, 10.1007/978-3-642-40811-3_23. hal-00833196

\section{HAL Id: hal-00833196 https://hal.science/hal-00833196}

Submitted on 12 Jun 2013

HAL is a multi-disciplinary open access archive for the deposit and dissemination of scientific research documents, whether they are published or not. The documents may come from teaching and research institutions in France or abroad, or from public or private research centers.
L'archive ouverte pluridisciplinaire HAL, est destinée au dépôt et à la diffusion de documents scientifiques de niveau recherche, publiés ou non, émanant des établissements d'enseignement et de recherche français ou étrangers, des laboratoires publics ou privés. 


\title{
Iterative Closest Curve: a Framework for Curvilinear Structure Registration Application to 2D/3D Coronary Arteries Registration
}

\author{
Thomas Benseghir $^{1,2 \star}$, Grégoire Malandain ${ }^{2 \star \star}$, and Régis Vaillant ${ }^{1}$ \\ ${ }^{1}$ GE-Healthcare, 78530 Buc, France \\ ${ }^{2}$ INRIA, 06900 Sophia Antipolis, France
}

\begin{abstract}
Treatment coronary arteries endovascular involves catheter navigation through patient vasculature. The projective angiography guidance is limited in the case of chronic total occlusion where occluded vessel can not be seen. Integrating standard preoperative CT angiography information with live fluoroscopic images addresses this limitation but requires alignment of both modalities.

This article proposes a structure-based registration method that intrinsically preserves both the geometrical and topological coherencies of the vascular centrelines to be registered, by the means of a dedicated curveto-curve distance pairs of closest curves are identified, while pairing their points. Preliminary experiments demonstrate that the proposed approach performs better than the standard Iterative Closest Point method giving a wider attraction basin and improved accuracy.
\end{abstract}

Keywords: registration, curvilinear structure, ICP, chronic total occlusion, CTO, coronary, X-ray, computed tomography angiography, CTA

\section{Introduction}

Percutaneous Coronary Intervention (PCI) is a minimally invasive procedure where a catheter is navigated through the patient vasculature in order to gain access to the pathology location. X-ray projective angiographic images allow the interventional cardiologists to visualize the vessels and devices and guide the endovascular intervention. In the case of Chronic Total Occlusion (CTO) contrast agent could not go through the occluded vessel which remains invisible in the angiography. Moreover, projective images do not allow 3D perception of the vessels whose local orientation may not be estimated correctly from the projective images, especially when the vessel is curved along the direction of the X-ray beam. On the other hand, preoperative CT angiography (CTA) provides

\footnotetext{
^ Asclepios project-team, Inria Sophia Antipolis - Méditerranée

** Morpheme project-team, Inria Sophia Antipolis - Méditerranée, I3S, UMR 7271, iBV, UMR 7277
} 
a static map of the entire vasculature, including occluded vessels and potential calcifications. Moreover, ambiguities due to the projective nature of angiography are not present in CTA. CTA acquisition is now standard in clinical practice before starting a difficult CTO case, due to the obtained understanding of the anatomical and lesion geometry is of unique value [12].

Integrating information extracted from CTA into fluoroscopic 2D images may facilitate guidance but necessitate a preliminary step of registration. The registration of the pre-operative data shall reach an accuracy of few millimeters at maximum in order to bring improved guidance to the operator. We explore feature-based registration approaches for their potential to bring the level of accuracy that we look for and also because the considered images present salient features to be used. Indeed the vessels are curvilinear structures which can be conveniently segmented in both CTA and fluoroscopic images made during an injection of contrast agent.

Features are represented by centerlines, which correspond to the curves following the vessel center, sometimes with radius information or perpendicular vessel cross-section. Leveraging curvilinear structures for solving registration problems has been tested in multiple medical fields: coronary [8] or cerebral [13] angiograms, liver images [3], [6] or in other applications [11], for diagnosis, planning and guidance purposes.

Several methods can be used to match two sets of features. Besl and McKay developed the Iterative Closest Point (ICP) algorithm [2] that is applicable to large frameworks, considering features as set of points. The algorithm is formed of three consecutive steps which are iterated: (i) Pair each point of a data to its closest in the model; (ii) Compute the transformation minimizing the mean square error between the paired points; (iii) Apply the transformation to the data and go back to (i). The success of the algorithm depends a lot on pairing and therefore on initial pose estimation. Thus, many variants have been introduced to improve the pairing construction. In [5], ICP is embedded into an expectation maximization framework, which allows multiple correspondences. Several other variants are compared in [10], all aiming to better build the pairings in order to improve registration robustness and accuracy.

Another class of methods, such as [13], formulate this problem as a matching measure optimization process in the set of admissible transformations. However, the energy measure to be optimized may have multiple local minima. Hierarchical approaches (gradually increasing searched transformation complexity) or combination of different optimization methods [9] partially help dealing with this issue. These local minima of the matching metric mainly come from the underlying point-to-point distance, and are thus due to pairing done without respecting the structural coherency. To improve this, [8] propose a hybrid metric between augmented points, including local structural information such as local direction, involving Euclidean distance from point to point and similarity properties of the iconic features. This improves the similarity of matched points, but is still inefficient to ensure a global coherency between the structures to be matched. 
Global coherency has been addressed in [3], [6] and [11] as a graph matching problem, where graph nodes (corresponding to vessel bifurcations or endpoints) are matched. However, such information may be unreliable in the aimed application because of the inherent characteristics of the $2 \mathrm{D}$ projective images (projection may induce error in bifurcation localization and introduces spurious ones by superposition of two vessels) and the potential segmentation errors.

We propose then to introduce a structure-based registration method, inspired by the ICP algorithm. It is dedicated to curvilinear structures, hence called Iterative Closest Curve, or ICC. Its main feature is that the built pairings conform with the vasculature structure of the images to be matched.

\section{Iterative Closest Curve Method}

The ICC-algorithm mimics the ICP algorithm introduced in [2], but with curves being considered instead of points. An intuitive notion of a curve allows one to understand the ICC framework and its capacity to preserve topological coherency during the registration process. Let the data $\mathcal{C}$ be a set of curves that can be registered to a model $\mathcal{X}$. The internal representation of $\mathcal{C}$ and $\mathcal{X}$ can be of any form but must allow one to extract curves from it.

The closest curve definition of an individual data curve $C \in \mathcal{C}$ in a model $\mathcal{X}$ is based upon a curve-to-curve distance $d$. We denote $X_{C}$ the closest curve of $C$ in $\mathcal{X}$ that satisfies

$$
X_{C}=\underset{X \in \mathcal{X}}{\operatorname{argmin}} d(C, X) .
$$

Contrary to standard closest point pairing, the resulting pairings insure topological and geometrical coherence since a curve is paired to another one.

Following the ICP framework we now define the best transformation $\hat{T}$ in a sense of least square curve registration:

$$
\hat{T}=\underset{T \in \Omega}{\operatorname{argmin}} \sum_{C \in \mathcal{C}} d^{2}\left(T(C), X_{C}\right)
$$

where $\Omega$ is the set of admissible transformations.

The ICC algorithm consists of the iteration of three steps:

1. Pair each curve $C$ in the set $\mathcal{C}$ to its closest curve $X_{C}$ in the set $\mathcal{X}$ (Eq. 1 )

2. Compute the transformation $\hat{T}$ minimizing the mean square error between the paired curves (Eq. 2)

3. Apply the transformation $\hat{T}$ to the set $\mathcal{C}$ and go back to 1 until convergence.

Now we have provided a framework for general curvilinear structure registration, some steps must be described to implement the ICC algorithm.

Data and model. The data $\mathcal{C}$ is defined as a set of $N_{\mathcal{C}}$ curves $\left(C_{k}\right)_{k=1 . . N_{\mathcal{C}}}$ of $\mathbb{R}^{3}$ representing the centerlines of a coronary vessel tree. These curves are traced from distal points to a common root point insuring a global coherence along an entire vessel during pairing. Concerning the model $\mathcal{X}$, curvilinear features are 
obtained from a 2D image by using a Hessian-based method [7] and then connected to obtain a non-directed graph structure. Therefore, $\mathcal{X}$ is composed of edges (centerline curves in $\mathbb{R}^{2}$ ) and nodes that are either extremities or bifurcations. Because of the projective nature of the image, detected bifurcations may be due to either a 3D bifurcation projection or a superimposition of two distinct vessels. No attempt to distinguish those cases has been attempted which may lead the graph to contain cycles.

Curve-to-curve distance. In the case of $3 \mathrm{D} / 2 \mathrm{D}$ registration, the distance $d$ in Eq. 1 deals with curves in different spaces $\left(\mathbb{R}^{3}\right.$ and $\left.\mathbb{R}^{2}\right)$. This problem can be overcome by applying a projective operator $P$ (given by system calibration) to the $3 \mathrm{D}$ curves when it is necessary. In practice, it is convenient to work with polygonal curves $C:[1, n] \rightarrow \mathbb{R}^{3}$ and $X_{C}:[1, m] \rightarrow \mathbb{R}^{2}$, constructed by linking $n$ points $\{C(p), p \in\{1 \ldots n\}\}$ (resp. $m$ and $\left\{X_{C}(p), p \in\{1 \ldots m\}\right\}$ ). A generic setto-set distance based on these construction points, e.g. the Hausdorff distance, can be considered but does not take into account the curvilinear structure of the data. The Fréchet distance between continuous polygonal curves, which has been addressed in [1], intrinsically takes into account the topological structures of curves. This distance respects the ordering of points along curves but is computationally expensive. A faster discrete version presented in [4] called the "coupling distance" gives an upper bound of the Fréchet distance by minimizing the distance between coupled points for all possible couplings. A coupling is a sequence of $Q$ pairs of points $\left(C(\gamma(1)), X_{C}(\lambda(1))\right), \ldots,\left(C(\gamma(Q)), X_{C}(\lambda(Q))\right)$ where the dummy variable $Q$ is ranged between $\max (n, m)$ and $(n+m)$ and $\gamma($ resp. $\lambda)$ is a non-decreasing surjection from $1 \ldots Q$ to $1 \ldots n$ (resp. to $1 \ldots m$ ) called a reparameterization mapping.

We derive from the Fréchet distance [4] the distance $d$ defined as

$$
d\left(C, X_{C}\right)=\sqrt{\min _{Q, \lambda, \gamma} \sum_{i=1 \ldots Q}\left\|P(C(\gamma(i)))-X_{C}(\lambda(i))\right\|^{2}} .
$$

These surjective mappings ensure that the summation is done over the complete set of points, forming the discrete curves. We imposed them to be nondecreasing to take into account the order of points along curves and because the pairing strategy defined below gives a scan sense direction along the curves.

Curves pairing. Eq. 1 requires to compute the Fréchet distance for all possible curves $X$ in the graph $\mathcal{X}$. A curve in $\mathcal{X}$ is defined as a path between two nodes, if it exists, without visiting twice the same edge. Yet, since the 2D graph may be noisy or complex the amount of possible curves can lead to a computationally explosive search. We propose to restrict the set of admissible curves by selecting candidates having their extremities in a neighborhood of the data curve extremities $C(1)$ and $C(n)$ projections. In practice, we identify edges (and nodes) in the vicinity of both $P(C(1))$ and $P(C(n))$ and construct the shortest path between them in the graph. If no $2 \mathrm{D}$ structure can be found close to the vessel distal part, a recursive shortening mechanism of the 3D curve is implemented to deal with topological differences between the data and the model. The shortest path is built upon distances along curves as edge weights. 
Transformation computation. Any optimization procedure can potentially be used to solve Eq. 2. Here we choose to take advantage of the underlying point-to-point pairing induced by the coupling realizing the Fréchet distance in Eq. 3. Indeed, for each curve $C_{k}$ we have a couple of reparameterization function $\gamma_{k}$ and $\lambda_{k}$ and a dummy variable $Q_{k}$ constituting point pairings between $\mathcal{C}$ and $\mathcal{X}$ that can be grouped as a single notation $\gamma, \lambda$ and $Q$. Then Eq. 2 can be rewritten as

$$
\hat{T}=\underset{T \in \Omega}{\operatorname{argmin}} \sum_{k=1}^{N_{\mathcal{C}}} \min _{Q_{k}, \lambda_{k}, \gamma_{k}} \sum_{i=1}^{Q_{k}}\left\|P\left(T\left(C_{k}\left(\gamma_{k}(i)\right)\right)\right)-X_{C_{k}}\left(\lambda_{k}(i)\right)\right\|^{2} .
$$

We propose to solve this least square problem by alternatively optimizing the transformation $T$ and the coupling variables $\gamma, \lambda$ and $Q$. For a given transformation $T$ finding the best coupling variables is simply obtained by computing the Fréchet distance. Coupling variables allows one to construct point pairing between set $\mathcal{C}$ and $\mathcal{X}$. Finding the best transformation is then equivalent to a least square alignment between two point sets with a given correspondence between points. We compute iteratively: (a) point-pairing optimization by Fréchet distance (between paired curves, the curve pairing being unchanged in this transformation computation loop); (b) transformation optimization at a fixed pointpairing; (c) applying the transformation to paired curves. This transformation optimization in ICC is thus equivalent to an "ICP"-like algorithm where closest point pairing is replaced by the Fréchet pairing at a given curve-to-curve pairing.

CTO handling. One major concern in registration is the topological difference between the data and the model. In the case of CTO, a part of a vessel or even the whole vessel is not visible in the $2 \mathrm{D}$ image, while it is present in the $3 \mathrm{D}$ CTA. This problem is solved in two different ways in the ICC algorithm. On the one hand, as mentioned in the curve pairing process, if no compatible curve can be found in the neighborhood of a vessel extremity we recursively shorten the $3 \mathrm{D}$ vessel. This approach can deal with missing parts of a vessel due to either CTO or lack of contrast leading to misdetection of $2 \mathrm{D}$ vessels. On the other hand, we implemented standard robust transformation estimators based on a loop of transformation optimization and pairing rejection based on a study of the residual error distribution. We introduce robust estimators in transformation computation at the ICC level (step 2.) by rejecting curve-pairings, but also inside the transformation computation when computing least square alignment (step b.) by rejecting point-pairings. The curve outlier rejection aims to deal with entire missing curves and point rejection with noisy detection.

\section{Results}

The goal of this result section is to give an example of applicability of the Iterative Closest Curve algorithm and prove its potential with respect to the standard Iterative Closest Point method. To this end we use four real data cases coming from three patients including one Chronic Total Occlusion (CTO) and one stenosis patient. One case is composed of a $3 \mathrm{D}$ segmentation of the left coronary tree 
(extracted via a commercial product), a fluoroscopic image and a manual pose estimation constituting the ground truth. Two cases were built from the CTO patient at different angulations. The sequences have been collected following standard clinical procedures and as such did not imply any additional procedural steps for the patient.

First, we want to highlight the structural coherency brought by the ICC methodology with respect to ICP in Fig. 1 and 2. For the same initial position (projected in blue) pairing are shown by segments linking data projection points and their paired corresponding points in the image. Fig. 1 presents the lack of coherence induced by closest point pairing along vessels. A single vessel in 3D presents different "jumps" in its pairing and is matched to multiple non-connected 2D detected curves. On the contrary, the ICC approach create a curve-to-curve pairing and the Fréchet distance imposes order coherence of the underlying point pairing along the two curves. As shown in Fig. 2, even if the pairing do not correspond to the expected one it seems more realistic. From the initial position presented in blue we run both ICC and ICP, leading to the results presented in Fig. 3. While the ICP algorithm gets stuck at a position where multiple vessels in $3 \mathrm{D}$ are transversally crossing $2 \mathrm{D}$ detected curves, the ICC algorithm converges to an acceptable 3D pose.
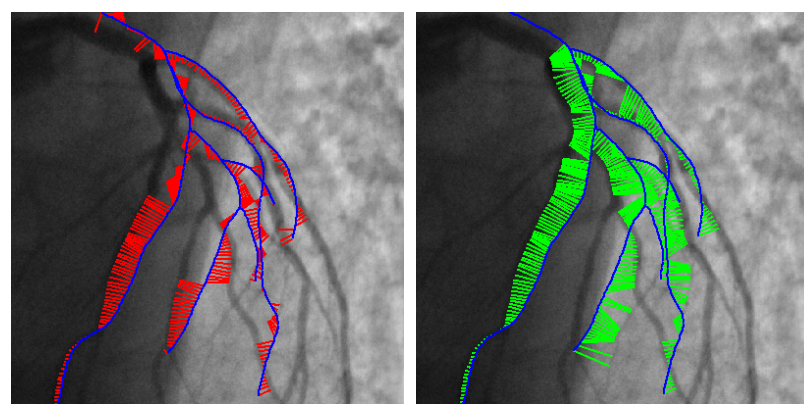

Fig. 1. Pairing obtained Fig. 2. Pairing obtained Fig. 3. ICC (green) and ICP with the ICP algorithm with the ICC algorithm

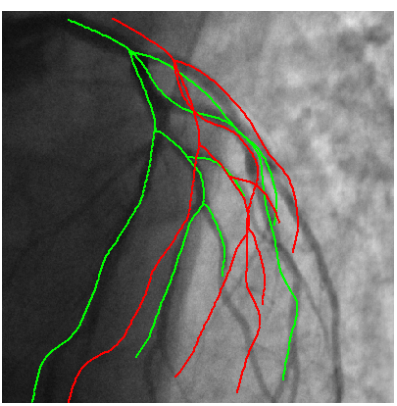

(red) registration results

Secondly, to confirm the robustness of ICC with respect to rotations we evaluate the registration algorithms on 100 random perturbations of the ground truth pose (manually determined). The random 3D displacement has been applied to the coronary tree and a step of alignment between the 3D root node and its detection in the 2D image, i.e. a translation, has been applied. This later step is an initialization step that seems realistic either in a manual or automatic way, thus only rotation pertubations have been investigated. We conducted experiments on three ranges of angular perturbation: 5, 10 and 15 degrees.

We assessed the registration error by the means of the Mean Projective Error (MPE); that is the average residual distance between projected 3D points after registration with respect to their ground truth counterparts. Fig. 4 displays the 


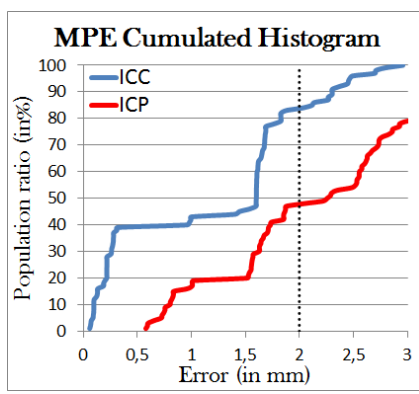

\begin{tabular}{|c|c|c|c|c|c|c|c|}
\hline \multirow{2}{*}{ Case } & \multirow{2}{*}{ MPE } & \multicolumn{6}{|c|}{$5^{\circ}$ rotation $10^{\circ}$ rotation $15^{\circ}$ rotation } \\
\hline & & ICC & ICP & ICC & ICP & ICC & ICP \\
\hline \multirow[b]{2}{*}{1} & mean & 1.4 & 2.1 & 3.2 & 5.0 & 6.9 & 9.3 \\
\hline & $90 \%$ & 3.0 & 3.7 & 7.4 & 10.3 & 15.6 & 18.8 \\
\hline \multirow{2}{*}{2} & mean & 1.3 & 2.3 & 2.0 & 3.9 & 4.2 & 8.3 \\
\hline & $90 \%$ & 3.0 & 4.4 & 4.3 & 8.1 & 9.3 & 16.8 \\
\hline \multirow{2}{*}{3} & mean & 1.7 & 3.4 & 4.6 & 6.9 & 7.1 & 10.6 \\
\hline & $90 \%$ & 3.9 & 6.6 & 8.7 & 13.9 & 12.4 & 21.2 \\
\hline \multirow{2}{*}{4} & mean & 1.3 & 1.8 & 2.6 & 4.3 & 7.5 & 8.7 \\
\hline & $90 \%$ & 3.3 & 3.6 & 6.2 & 8.6 & 16.5 & 18.2 \\
\hline
\end{tabular}

Fig. 4. Statistics on Mean Projected Error (MPE). Left: the cumulated MPE for case 1 obtained with 100 random rotations for $5^{\circ}$ of rotation. Right: mean and 90th percentile of the MPE distribution for ICC and ICP algorithm

cumulative MPE histogram for one patient and one angle (5 degrees). It demonstrates that the ICC (blue curve) performs better than the ICP (red curve). For example, a $2 \mathrm{~mm}$ MPE is reached in only $50 \%$ of tested positions for ICP and in more than $80 \%$ for ICC.

Fig. 4 (right) also shows a table capturing the behavior of ICC and ICP algorithms for the whole dataset by computing the average and ninetieth percentile of MPEs. ICC obtains lower values for both indicators, suggesting that ICC can be more accurate that ICP, and that point based methods can benefit from integration of global structural information.

Current implementation runs in around five seconds on an Intel CORE i5 cadenced at $1.5 \mathrm{GHz}$.

\section{Conclusion}

We proposed in this paper a general registration method for curvilinear structure taking into account geometrical and topological coherency of the data. We exemplified the ICC method with a 2D/3D registration implementation applied to coronary artery registration. By introducing curve-to-curve pairing and distance, we showed that ICC algorithm overpassed the standard ICP algorithm and significantly improved the resulting pairings. The improvement in the pairings has been visually evaluated in test experiments. We also estimated the improvement by comparing the mean projection error obtained with our approach to the reference ICP method.

For future work, the capacity of this algorithm to handle transformation scheme involving non-rigid deformations needs to be evaluated. Indeed, we observed in our test application that the rigid transformation is insufficient to describe the changes supported by the anatomy between the pre- and the peroperative imaging situation. Additional outlier rejection mechanisms shall also be added to extend further the attraction basin. 


\section{References}

1. Helmut Alt and Michael Godau. Measuring the resemblance of polygonal curves. In Proceedings of the eighth annual symposium on Computational geometry, SCG '92, pages 102-109, New York, NY, USA, 1992. ACM.

2. P.J. Besl and N.D. McKay. A method for registration of 3-D shapes. IEEE Transactions on Pattern Analysis and Machine Intelligence, 14(2):239-256, February 1992.

3. Arnaud Charnoz, Vincent Agnus, Grégoire Malandain, Clément Forest, Mohamed Tajine, and Luc Soler. Liver registration for the follow-up of hepatic tumors. In J. Duncan and G. Gerig, editors, Proceedings of MICCAI'05, volume 3750 of LNCS, pages 155-162, Palm Springs, California, 2005. Springer.

4. Thomas Eiter and Heikki Mannila. Computing discrete Fréchet distance. Technical Report CD-TR 94/64, Christian Doppler Laboratory for Expert Systems, TU Vienna, Austria, 1994.

5. Sébastien Granger, Xavier Pennec, and Alexis Roche. Rigid point-surface registration using an EM variant of ICP for computer guided oral implantology. In W.J. Niessen and M.A. Viergever, editors, 4th Int. Conf. on Medical Image Computing and Computer-Assisted Intervention (MICCAI'01), volume 2208 of LNCS, pages 752-761, Utrecht, The Netherlands, October 2001.

6. Martin Groher, Darko Zikic, and Nassir Navab. Deformable 2D-3D registration of vascular structures in a one view scenario. IEEE Transactions on Medical Imaging, 28(6):847-60, June 2009.

7. K. Krissian, G. Malandain, N. Ayache, R. Vaillant, and Y. Trousset. Model-based detection of tubular structures in 3D images. Computer Vision and Image Understanding, 80(2):130-171, 2000.

8. C. Metz, M. Schaap, S. Klein, N. Baka, L. Neefjes, C. Schultz, W. Niessen, and T. van Walsum. Registration of $3 \mathrm{D}+\mathrm{t}$ coronary CTA and monoplane $2 \mathrm{D}+\mathrm{t}$ X-ray angiography. IEEE Transactions on Medical Imaging, 32(5):919-31, 2013.

9. David Rivest-Henault, Hari Sundar, and Mohamed Cheriet. Nonrigid 2D/3D registration of coronary artery models with live fluoroscopy for guidance of cardiac interventions. IEEE Transactions on Medical Imaging, 31(8):1557-72, August 2012.

10. S. Rusinkiewicz and M. Levoy. Efficient variants of the ICP algorithm. In 3-D Digital Imaging and Modeling. IEEE, 2001.

11. Eduard Serradell, Przemyslaw Glowacki, Jan Kybic, Francesc Moreno-Noguer, and Pascal Fua. Robust Non-Rigid Registration of 2D and 3D Graphs. In Conference on Computer Vision and Pattern Recognition, 2012.

12. Pinak B Shah. Management of coronary chronic total occlusion. Circulation, 123(16):1780-1784, 2011.

13. Hari Sundar, Ali Khamene, Chenyang Xu, Frank Sauer, and Christos Davatzikos. A novel 2D-3D registration algorithm for aligning fluoro images with $3 \mathrm{D}$ pre-op CT/MR images. In Medical Imaging, volume 6141, page 61412K. SPIE, 2006. 